УДК: 004.91:33

https://doi.org/10.36906/AP-2020/46

\title{
ИНФОРМАЦИОННАЯ СИСТЕМА ДЛЯ АВТОМАТИЗАЦИИ ПРОЦЕССА ДЕЯТЕЛЬНОСТИ МЕНЕДЖЕРА АГЕНТСТВА НЕДВИЖИМОСТИ
}

\author{
Юшков В. С. \\ Московский педагогический государственный \\ университет (Анапский филиал) \\ 2. Aнапа, Россия
}

Аннотация. Развитие информационных технологий на современном этапе развития науки и техники позволяет их внедрять во все сферы жизнедеятельности человека. В данной статье представлен программный продукт, разработанный для менеджера агентства недвижимости. Внедрение данного продукта позволит сократить время на работу с клиентом, свести к минимуму документооборот, переведя его в электронный вариант.

Ключевые слова: менеджер, автоматизированная информационная система, риэлтор, недвижимость, язык программирования.

Автоматизированная информационная система (АИС) представляет собой сложный комплекс, который включает в себя совокупность различных программно-аппаратных платформ, универсальных и специализированных приложений различных разработчиков, интегрированных в единую информационно-однородную систему, которая помогает решать задачи каждой конкретной организации [1].

Внедрение АИС обусловлено следующими основными целями:

- рост эффективности компании за счет появления новых возможностей;

- повышение внутриорганизационной производительности; улучшение обслуживания клиентов; улучшение управления бизнесом [2];

Деятельность агентства недвижимости, характеризуется следующими особенностями:

- основным направлением деятельности является реализация недвижимости, представленной собственными новостройками;

- использование широкого спектра форм расчетов, применение программ ипотеки и кредитования;

- многочисленный штатный состав сотрудников, требующий качественной информационной поддержки.

Для повышения производительности труда требуется внедрить автоматизированную информационную систему в агентство недвижимости. С этой целью был разработан программный продукт, позволяющий автоматизировать деятельность менеджера.

Разработка автоматизированной информационной системы начинается с построения модели базы данных. Для работы с SQLite базой данных было выбрано программное обеспечение "DB Browser for SQLite". Данное программное обеспечение позволяет работать с базой данных в удобном графическом интерфейсе и создать базу данных, полностью настроив ее для дальнейшей работы и возможности создания на ее основе графического интерфейса приложения.

В качестве структуры базы данных выступают две таблицы. Первая таблица отвечает за клиентскую базу, в которой хранится информация о фамилии, имени и отчестве, а также о 


\section{СОВРЕМЕННОЕ ПРОГРАММИРОВАНИЕ}

III Международная научно-практическая конференция

контактах клиента. Далее эти поля будут использоваться для информации о заявках на объекты с помощью внешнего ключа, чтобы избежать дублирования данных и перегрузки базы данных.

Для этого создается таблица Users, в которой формируются три поля. Первое поле id_User является числовым первичным ключом и имеет свойства автоинкрементирования, позволяющее автоматически увеличивать значение на одну единицу относительно предыдущей строки. Второе поле FIO является текстовым и необходимо для хранения информации о фамилии, имени и отчестве клиента. Третье поле Contacts также является текстовым и используется для хранения в нем информации о контактах клиента.

Вторая таблица же включает в себя информацию о заявках на объекты. Объект имеет информацию о связанном с ним клиенте, типе объекта, информации о самом объекте, его цене и статусе. Так как не имеет смысла создавать несколько таблиц для объектов одного типа, но с разным типом заявок, создается поле типа, которое делит одну таблицу на разделы, такие как аренда, сдача, покупка, продажа.

Для реализации такой таблицы создается таблица Hous, имеющая 6 полей. Первое поле id_Hous является числовым первичным ключом и имеет свойства автоинкрементирования. Второе поле, это Туре, которое является текстовым и отражает в себе тип заявки. Оно необходимо для деления на разделы, о которых упоминалось ранее. Третье поле Price содержит в себе цену, которую клиент готов потратить на свою заявку. Четвертое поле текстовое и называется Information. Оно хранит в себе всю побочную информацию об объекте и заявке, такую как описание объекта, какие-то условия клиента и другое. Пятое поле Seller, это числовой внешний ключ и несет информацию о клиенте, связывая строку с другой строкой из таблицы Users. Шестое поле Status необходимо для отображения статуса заявки, а конкретнее - для отражения актуальности.

Структура и запросы, необходимые для создания данных таблиц в программе “DB Browser for SQLite", отражены на рисунках 1-2.

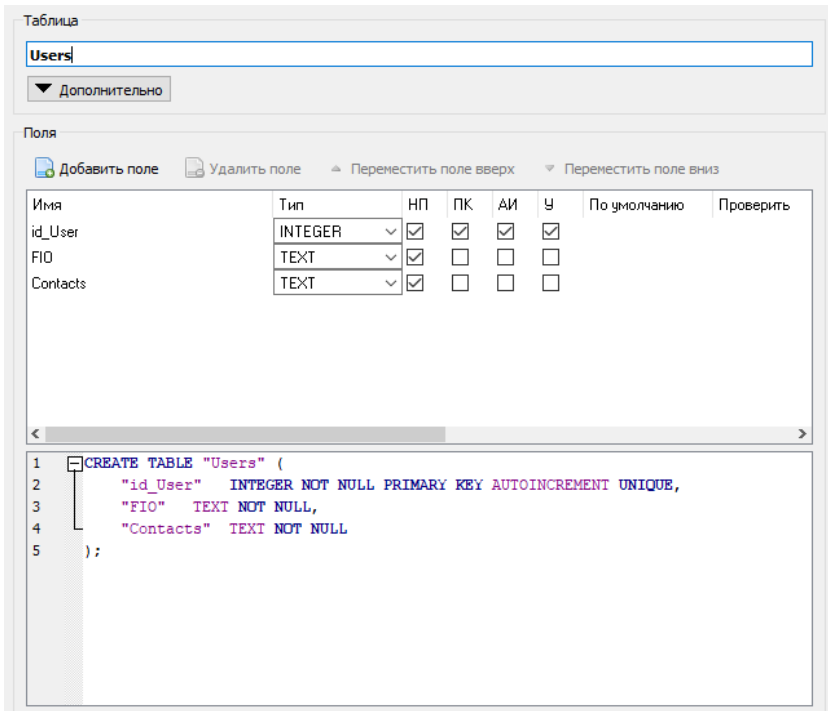

Рис. 1. Создание таблицы Users

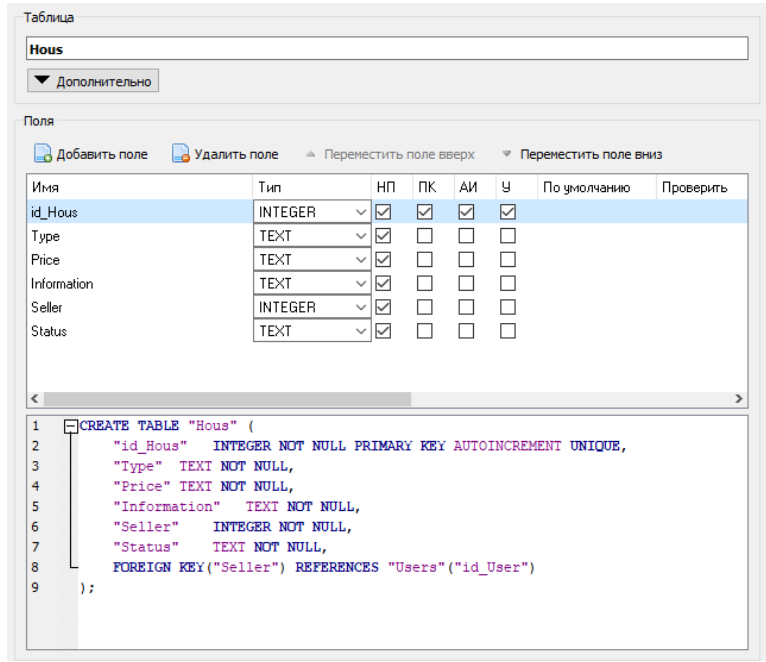

Рис. 2. Создание таблицы Hous

На этом разработка базы данных завершена. Далее необходимо разработать графический интерфейс и подключить базу данных к нему.

В первую очередь создается проект Windows Forms С\# в среде разработки Visual Studio 2019. После этого необходимо подключить библиотеку SQLite. Для этого нужно зайти 


\section{СОВРЕМЕННОЕ ПРОГРАММИРОВАНИЕ}

\section{III Меякднародная научно-практическая конференция}

в управление пакетами NuGet, которое интегрировано в нашу среду разработки, и найти там "System.Data.SQLite", после чего нажать установить, и на этом процесс добавления библиотеки завершен [3-4].

После чего необходимо создать форму авторизации (рис. 3), которая содержит два текстовых поля в виде элемента TextBox и кнопку в виде элемента Button. Второе текстовое поле, необходимое для ввода пароля, имеет свойство PasswordChar pавное*. Это необходимо для скрытия символов, вводимых в данное поле. Кнопка выполняет функцию проверки введенных данных, и, если они верные, то запускает приложение и закрывает форму авторизации. В иных случаях выводит сообщение о неверно введенных данных [5].

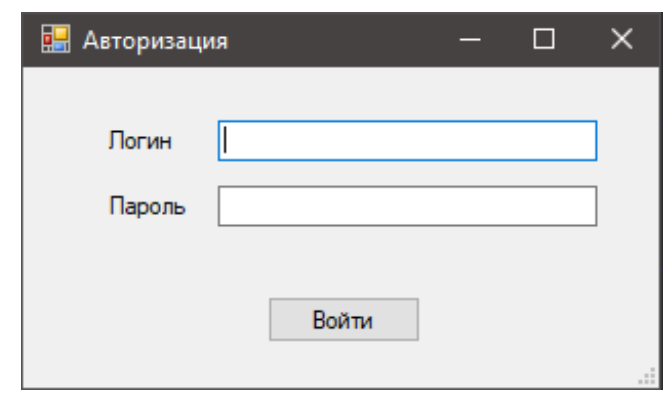

Рис. 3. Форма авторизации

При разработке интерфейса учитывались основные требования, предъявляемые к работе менеджера, поэтому были разработаны 4 вкладки, которым присвоены имена:

1) информация о клиентах;

2) недвижимость купля-продажа;

3) недвижимость аренда;

4) куплено/продано;

На рисунке 4 представлена первая вкладка.

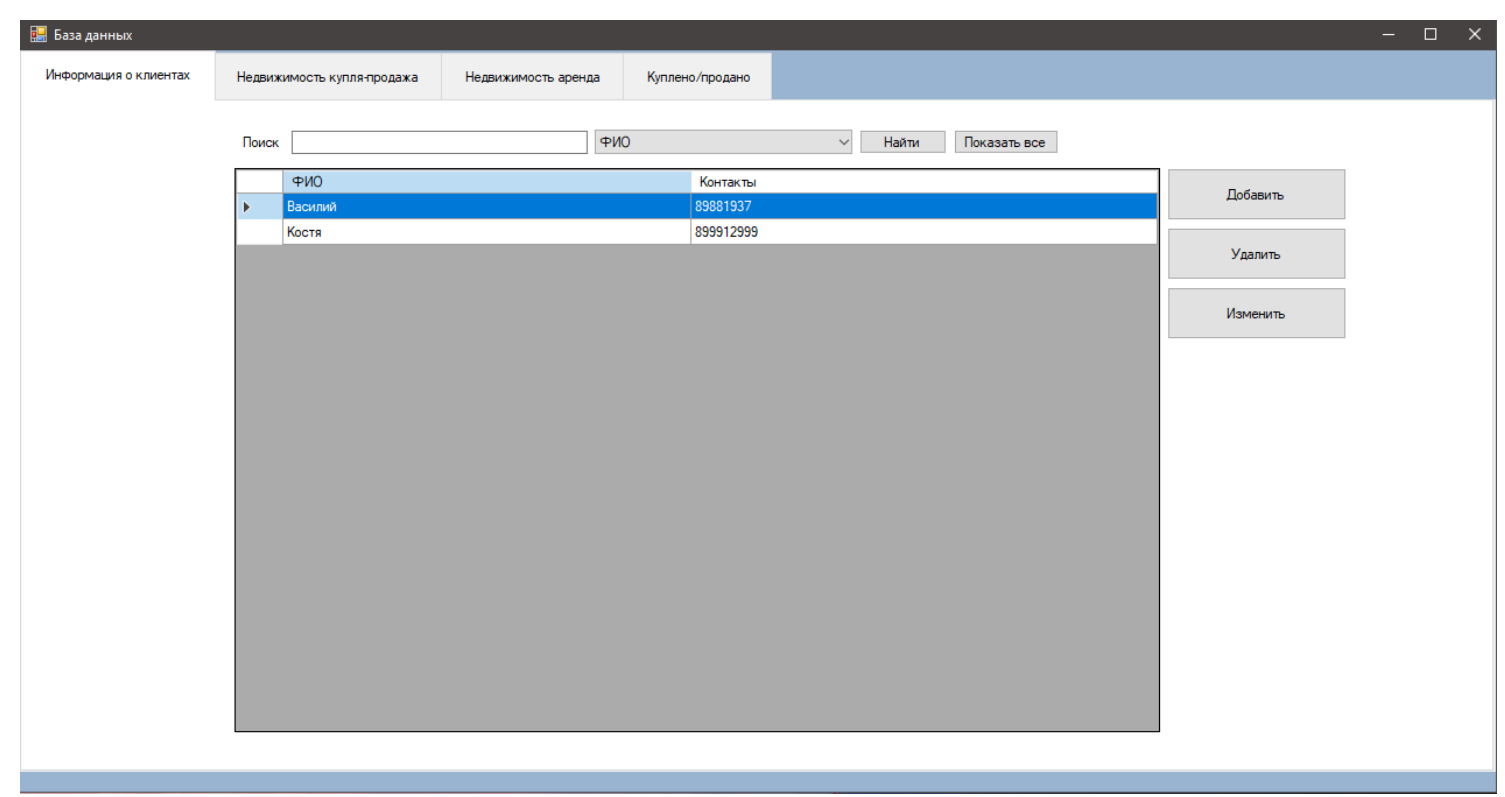

Рис. 4. Вкладки элемента TabControl

На первой вкладке «Информация о клиентах» находятся элементы:

1. Таблица DataGridView; 


\section{СОВРЕМЕННОЕ ПРОГРАММИРОВАНИЕ}

\section{III Международная научно-практическая конференция}

2. Кнопка Button для добавления записей;

3. Кнопка Button для удаления записей;

4. Кнопка Button для изменения записей;

5. Текстовое поле ТехtВох для ввода поисковых запросов;

6. Выпадающий список ComboBox для выбора, по каким данным производить поиск;

7. Кнопка Button для поиска;

8. Кнопка Button для отображения всех данных без поиска и сортировки;

Вторая вкладка имеет другой набор элементов, позволяющих менять выборку и комбинировать это с поисковыми запросами (рис. 5). На ней находятся элементы:

1. Таблица DataGridView;

2. Выпадающий список ComboBox меняющий выборку по типу объекта;

3. Кнопка Button для добавления записей;

4. Кнопка Button для удаления записей;

5. Кнопка Button для изменения записей;

6. Кнопка Button для изменения статуса объекта на проданный/купленный;

7. Текстовое поле ТеxtВох для ввода поисковых запросов;

8. Выпадающий список ComboBox для выбора, по каким данным производить поиск;

9. Кнопка Button для поиска;

10. Кнопка Button для отображения всех данных без поиска и сортировки;

11. Данная вкладка изображена на рисунке 5.

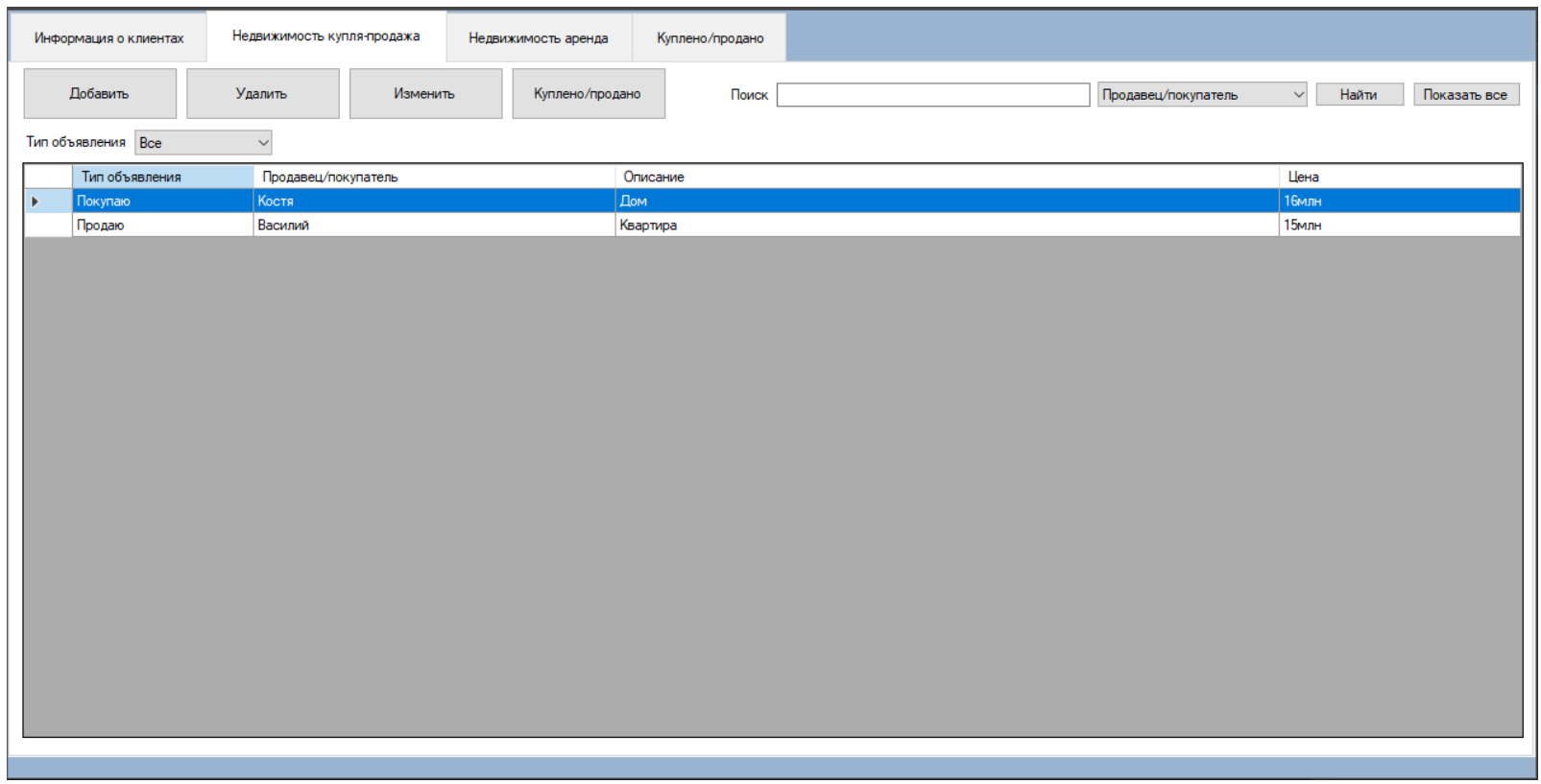

Рис. 5. Элементы второй вкладки TabControl

Третья вкладка имеет тот же набор элементов, что и второй, за исключением названия кнопки изменения статуса, а также набора вариантов выбора в выпадающем списке, предназначенном для изменения выборки. А так же, здесь выводятся в таблицу данные, связанные с арендой, а не куплей-продажей. И все запросы, связанные с элементами управления, так же настроены на работу с данными объектов аренды. В остальном набор и расположение элементов не отличается от второй вкладки. Третья вкладка изображена на рисунке 6. 


\section{СОВРЕМЕННОЕ ПРОГРАММИРОВАНИЕ}

\section{III Международная научно-практическая конференция}

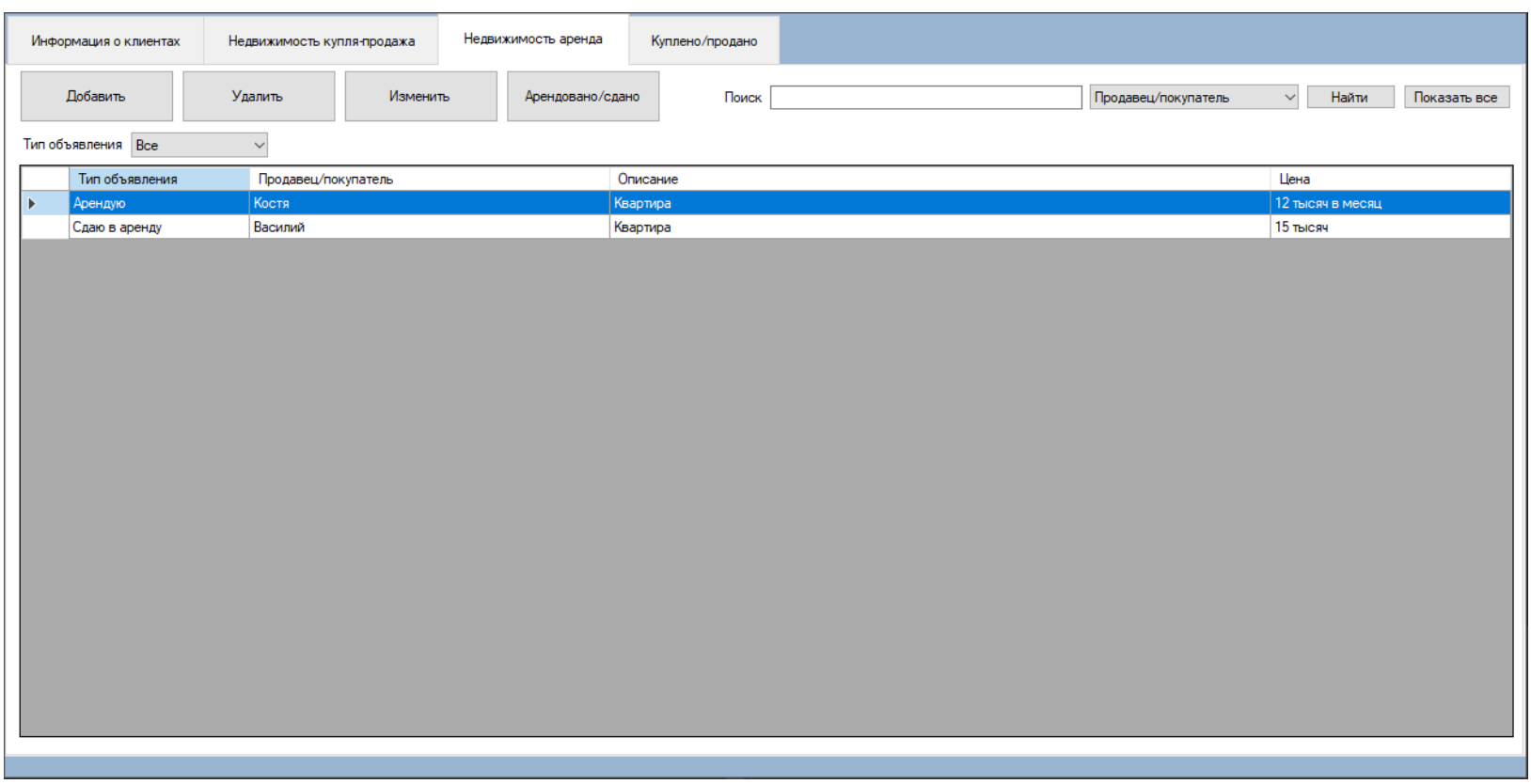

Рис. 6. Элементы третьей вкладки TabControl

Четвертая вкладка необходима для просмотра объектов с завершенным статусом (рис. 7). Данная вкладка включает в себя следующие элементы:

1. Таблица DataGridView;

2. Кнопка Button для удаления записей;

3. Текстовое поле ТеxtВox для ввода поисковых запросов;

4. Выпадающий список ComboBox для выбора, по каким данным производить поиск;

5. Кнопка Button для поиска;

6. Кнопка Button для отображения всех данных без поиска и сортировки.

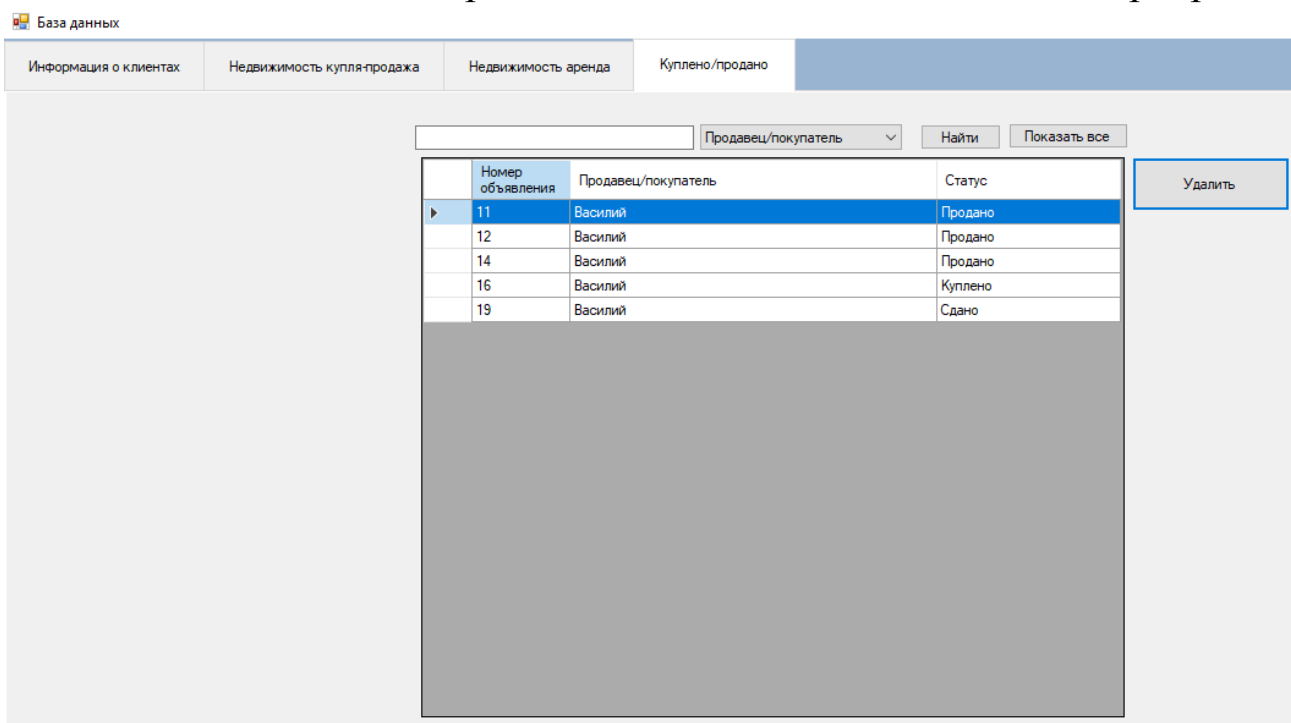

Рис. 7. Элементы четвертой вкладки TabControl

В первую очередь была создана функция ShowGrid(). Это универсальная функция, аргументами которой являются запрос к базе данных, и ссылка на объект DataGridView в котором необходимо отобразить результат запроса. Данная функция создает внутри себя объект DataTable, после чего создается объект SQLiteDataAdapter, в который передается 
запрос к базе данных и объект SQLiteConnection, созданный ранее для подключения к базе данных. Далее с помощью метода Fill() объекта SQLiteDataAdapter заполняются данные в объект DataTable. После этого проверятся, есть ли строки в DataTable. Если они есть, то с помощью цикла все строки из DataTable добавляются в отчищенный DataGridView, который был передан функции в качестве аргумента. Если же строки в DataTable отсутствуют, то выводится сообщение о том, что запрос не выдал никакого результата. Для вывода данных используется объект MessageBox и его метод Show().

Первый вызов функции ShowGrid() происходит при загрузке формы для отображения данных в таблице на первой вкладке элемента TabControl. Для этого используется запрос “SELECT * FROM Users".

Далее вызов функции происходит при изменении активной вкладки в элементе TabControl. У данного элемента есть событие SelectedIndexChanged, которое срабатывает при изменении активной вкладке. Внутри данного события создается структура switch case, в которую передается свойство SelectedIndex объекта TabControl, имеющее в себе информацию о номере активной вкладки на данный момент. Далее для каждой вкладки создается запрос, результат которого будет отображаться при открытии каждой из вкладок.

Для первой вкладки — это запрос “SELECT * FROM Users”. Данный запрос возвращает все доступные значения из таблицы Users.

Для второй вкладки используется запрос "SELECT Hous.id_Hous, Hous.Type, Users.FIO, Hous.Information, Hous.Price FROM Hous LEFT JOIN Users ON Hous.Seller = Users.id_User WHERE Hous.Type like 'Продаю' AND Hous.Status like 'Доступно' OR Hous.Type like 'Покупаю' AND Hous.Status like 'Доступно'”. Этот запрос возвращает тип объекта, ФИО клиента, информацию об объекте, цену объекта из таблицы Hous и Users, a также делает выборку по типу объекта и статусу. В данном случае выборка выводит объекты со статусом «Доступно» и типом объекта «Покупаю» или «Продаю».

В третьей вкладке используется схожий запрос «SELECT Hous.id_Hous, Hous.Type, Users.FIO, Hous.Information, Hous.Price FROM Hous LEFT JOIN Users ON Hous.Seller = Users.id_User WHERE Hous.Type like 'Арендую' AND Hous.Status like 'Доступно' OR Hous.Type like 'Сдаю в аренду' AND Hous.Status like 'Доступно'». Данный запрос выводит те же данные, что и запрос для второй вкладки, за исключением того, что выборка по типу здесь имеет другие значения. Она возвращает значения с типом объекта «Сдаю в аренду» или «Арендую».

В четвертой вкладке запрос выглядит следующим образом: «SELECT Hous.id_Hous, Users.FIO, Hous.Status FROM Hous LEFT JOIN Users ON Hous.Seller = Users.id_User WHERE Hous.Status NOT LIKE 'Доступно'». Данный запрос возвращает данные о номере объекта, ФИО связанного с ним клиента и статус объекта. Выборка производится по статусу объекта и в данном случае выводит все записи, в которых статус отличается от «Доступно».

Кнопка «Добавление» создает объект Form2, и передает в него ссылку на DataGridView из данной вкладки, а также текущий запрос, который отображает данные в данной таблице. После вызывает новую форму с помощью метода ShowDialog(). Данная форма предоставлена на рисунке 8 . 


\section{СОВРЕМЕННОЕ ПРОГРАММИРОВАНИЕ}

\section{III Международная научно-практическая конференция}

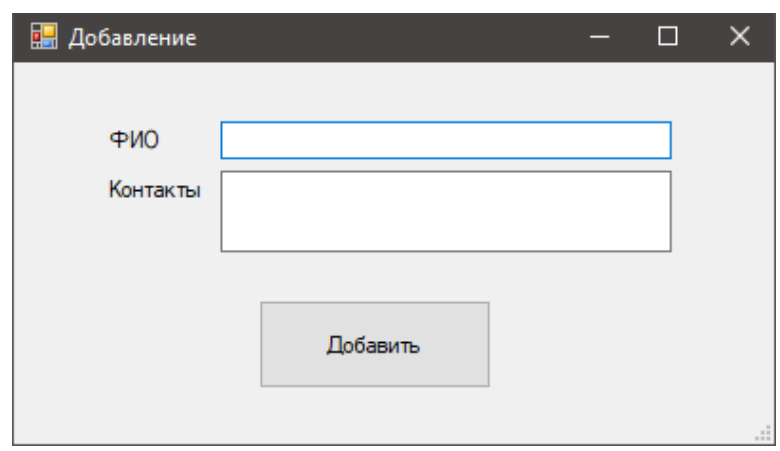

Рис. 8. Форма добавления

Данная форма имеет в себе два поля TextBox и одну кнопку Button. Так же при открытии данная форма подключается к базе данных таким же методом, как и основная форма приложения. Текстовые поля предназначены для ввода данных о клиентах. Кнопка необходима для выполнения запроса.

При нажатии на кнопку она считывает данные из текстовых полей и проверяет, не являются ли они пустыми с помощью сравнения данных из полей с данными, которые возвращает метод Empty объекта String. Если данные поля пустые, то выводится сообщение с просьбой заполнить поля. Если же они имеют данные, то формируется запрос в переменной типа String "INSERT INTO Users ('FIO', 'Contacts') values ('" + fio + "', '" + contact + "')" в котором переменные fio и contacts являются данными из текстовых полей. Далее создается объект SQLiteCommand, которому передается запрос и ссылка на объект SQLiteConnection. После чего вызывается метод ExecuteNonQuery() объекта SQLiteCommand, который выполняет запрос.

В конце обновляются данные в таблице DataGridView, ссылка на которую была передана при создании формы, и запрос для обновления был передан вместе с ней. Обновление происходит с помощью функции ShowGrid(). И после этого форма закрывается.

Далее рассмотрим кнопку «Изменить». Данная кнопка так же создает объект формы, и передает в него ссылку на DataGridView, а так же текущий запрос. После вызывает новую форму с помощью метода ShowDialog(). Данная форма предоставлена на рисунке 9.

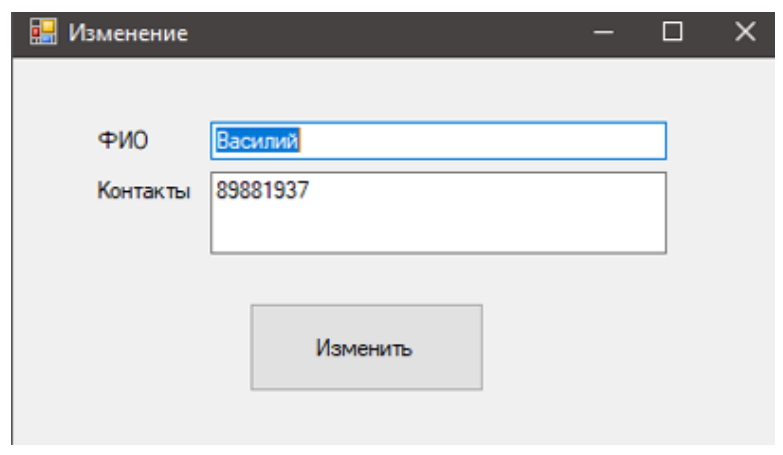

Рис. 9. Форма изменения

Основным отличием данной формы от формы добавления является то, что ссылку на объект DataGridView она использует сразу для заполнения данных в поля. При загрузке формы берутся данные из текущей выделенной записи в DataGridView и заполняются в текстовые поля на форме. После чего пользователь может как-либо их изменить, 
использовать кнопку «Изменить» для подтверждения изменений. Алгоритм кнопки не сильно отличается от кнопки для добавления.

При нажатии на кнопку изменения она так же считывает данные из текстовых полей и проверяет, не являются ли они пустыми с помощью сравнения данных из полей с данными, которые возвращает метод Empty объекта String. Если данные поля пустые, то выводится сообщение с просьбой заполнить поля. Если же они имеют данные, то формируется запрос в переменной типа String "UPDATE Users SET FIO = "' + textBox1.Text + "', Contacts = "" + textBox2.Text + "' WHERE id_User $=$ "' + DataGrid $[0$, DataGrid.CurrentRow.Index].Value.ToString ()$+$ "'» в котором переменные textBox1.Text и textBox2.Text являются данными из текстовых полей, а метод объекта DataGrid[0, DataGrid.CurrentRow.Index].Value.ToString() является ссылкой на номер клиента, данные которого необходимо изменить. Далее создается объект SQLiteCommand, которому передается запрос и ссылка на объект SQLiteConnection. После чего вызывается метод ExecuteNonQuery() объекта SQLiteCommand, который выполняет запрос.

В конце так же обновляются данные в таблице DataGridView, ссылка на которую была передана при создании формы, и запрос для обновления был передан вместе с ней. Обновление происходит с помощью функции ShowGrid(). И после этого форма закрывается.

Кнопку «Удалить». Данная кнопка при нажатии записывает в переменную типа String rowId номер записи, которая на данный момент выделена в DataGridView с помощью метода dataGridView1[0, dataGridView1.CurrentRow.Index].Value.ToString(). После чего создается объект SQLiteCommand, которому передается запрос «DELETE FROM Users WHERE id_User $="+$ rowId + "», который удаляет запись с номером записи rowId и ссылка на объект SQLiteConnection. После чего вызывается метод ExecuteNonQuery() объекта SQLiteCommand, который выполняет запрос. И обновляет данные с помощью функции ShowGrid().

Все кнопки «Удалить» на всех вкладках работают по тому же принципу, поэтому это описание универсальное и больше описания кнопки удаления не будет.

Рассмотрим способ работы элементов поиска. На всех вкладках они практически идентичные за исключением второй и третьей. Основой является текстовое поле, в которое записывается искомое значение. Так же выпадающий список, который содержит в себе все виды полей для вкладки, на которой он находится. И кнопка поиска.

При нажатии на кнопку поиска, считывается значение из текстового поля и если оно не является пустым, то записывается в переменную типа String. После чего строится конструкция switch case, в которой происходят изменения относительно значения выбранного в выпадающем списке. Для этого узнается индекс выбранного значения с помощью свойства SelectedIndex объекта ComboBox. После чего формируется запрос для выбранного вида поля, который сохраняется для дальнейшего использования при добавлении и изменении. Далее вызывается функция ShowGrid, в которую передается данный запрос и ссылка на объект DataGridView на данной вкладке.

Основным отличием для второй и третьей вкладки является тип выборки, которых может быть три. Это выборка всего, выборка только покупки или только продажи для второй вкладки, или выборка только сдачи в аренду или аренды для третьей вкладки. Осуществляется выборка с помощью элемента ComboBox и его события SelectedIndexChanged, которое срабатывает при изменении выбранного элемента из выпадающего списка. После чего с помощью структуры switch case и номера выбранного элемента выполняются запросы на выборку необходимых данных с помощью функции ShowGrid(). А так же запоминается значение текущей выборки для использования в поиске. 
С помощью значения выборки для поиска на второй и третьей вкладке структура switch case усложняется, добавляя по три запроса для каждого вида поля. И в зависимости от выбранной выборки и поля для поиска будет формироваться один итоговый запрос.

Для формирования запросов поиска используется структура WHERE «название поля» LIKE «искомое значение», а также логические выражения для комбинирования, такие как $\mathrm{AND}, \mathrm{OR}$. Для каждой вкладки берется изначальный запрос и к нему добавляется значения искомых полей и требуемые значения. А для второй и третьей вкладки добавляется еще и значения выборки, которая так же выполняется с помощью данной структуры. Для примера возьмем запрос поиска по всем записям купле-продажи «SELECT Hous.id_Hous, Hous.Type, Users.FIO, Hous.Information, Hous.Price FROM Hous LEFT JOIN Users ON Hous.Seller = Users.id_User WHERE Hous.Type like 'Покупаю' AND Hous.Status like 'Доступно' AND Users.FIO LIKE '\%" + find + "\%' OR Hous.Type like 'Продаю' AND Hous.Status like 'Доступно' AND Users.FIO LIKE '\%" + find + "\%'». Здесь поиск осуществляется по ФИО клиента, а выборка настроена на отображения и покупки и продажи объектов.

Кнопка «Показать все» используется для отображения первоначального состояния таблицы без поисковых запросов и отдельных типов выборки. На каждой вкладке данная кнопка вызывает функцию ShowGrid () с первоначальным запросом, который отображал данные при открытии данной вкладки.

Так же простой кнопкой является изменение вкладки статуса. Данные кнопки на второй и третьей вкладке считывают номер выделенной записи и ее статус, после чего с помощью проверки условия устанавливают завершенную версию текущего значения с помощью выполнения запроса методом ExecuteNonQuery () объекта SQLiteCommand.

Кнопки «Добавить» и «Изменить» для второй и третьей вкладки идентичны за исключением передаваемых в них ссылок и запросов, которые уже относятся к конкретной вкладке. Так же формы, которые они создают, отличаются лишь разными типами объектов, а в остальном являются идентичными.

Таким образом, разработанный программный продукт позволяет автоматизировать работу менеджера по продаже недвижимости, в частности, сократить время, затрачиваемое на оформление документов по объекту недвижимости; снизить ошибки, вызванные человеческим фактором. Простой интерфейс программы не требует дополнительного обучения менеджера, а, соответственно - затрат на обучение [6, 7].

\section{Литература}

1. Абросимова М. А. Информационные технологии в государственном и муниципальном управлении. М.: КноРус, 2013. 248 с.

2. Жданов С. А., Соболева М. Л., Алфимова А. С. Информационные системы. М.: Прометей, 2015. 302 с.

3. Белоногов Г. Г., Новоселов А. П. Автоматизация процессов накопления, поиска и обобщения информации. М.: Наука, 2017. 256 с.

4. Мезенцев К. Н. Автоматизированные информационные системы. М.: Academia, 2012. $176 \mathrm{c}$.

5. Юшков В. С. Несанкционированный доступ, как проявление информационного экстремизма // Глобализация научных процессов: сб. ст. по материалам международной научно-практической конференции. Иркутск, 2017. С. 87-90. 


\section{СОВРЕМЕННОЕ ПРОГРАММИРОВАНИЕ}

III Меякднародная научно-практическая конференция

6. Юшков В. С., Еременко Д. О. Влияние информационных технологий на современное общество XXI века // Образование, наука, культура: традиции и современность: материалы VIII Всероссийской научно-практической конференции. Анапа. 2018. С. 104-107.

7. Якушевский М. С., Юшков В. С., Якименко С. А. Применение автоматизированных систем управления в жизнедеятельности человека // Развитие интеллектуального потенциала молодежи Кубани Материалы: материалы Всероссийской научно-практической конференции, посвященной 20-летию Анапского филиала МПГУ. 2018. С. 155-160.

(СЮиков В. С., 2020 